\title{
Suicidio en el mundo: ¿puede un enfoque evolutivo explicar la relación entre tasas de suicidio y variables asociadas a calidad de vida? \\ Suicide worldwide: can the evolutionary perspective explain the relationship between suicide rates and quality of life variables?
}

Jorge Rodríguez R. ${ }^{1}$, Jaime Santander T. ${ }^{1}$, Alberto Aedo S. ${ }^{1}$ y Sebastián Robert B. ${ }^{2}$

\begin{abstract}
Introduction: Suicide is a complex phenomenon and seems to be paradoxical from an evolutionary standpoint, considering the multiple associated risk factors and the differences in suicide rates among countries. Suicide risks are usually attributed to economic hardship and low quality of life. However, some studies have shown an inverse relationship. Objective: To compare suicide rates reported for different countries to date with economic and quality of life indicators. Method: Suicide rates reported for 82 countries by the World Health Organization (WHO) were correlated with 2008 purchasing power parities (PPPs) and human development indexes (HDIs) published by the World Bank and the United Nations, respectively. Results: A positive correlation was observed between suicide rates, PPPs and HDIs ( $r=0.3601$ and $r=0.366$, respectively $p<0.01$ ). Conclusions: The positive correlation observed (i.e., suicide rates increased with economic and quality of life indicators) seem to be contradictory on the surface. We propose that interpreting these data from an evolutionary perspective could contribute to a deeper understanding of this phenomenon. Nevertheless, there are a myriad of factors that could be contributing to these results, thus further research is needed to understand suicide at a global scale.
\end{abstract}

Key words: Suicide, Economic Development, Biological Evolution, Quality of Life. Rev Chil Neuro-Psiquiat 2014; 52 (4): 243-249

\section{Introducción}

$\mathrm{E}_{\mathrm{a}}^{\mathrm{l}}$ suicidio es un fenómeno complejo, ligado a la naturaleza humana y que tiene representación global. Se estima que la tasa de mortalidad mundial por suicidio alcanza los 14,5 por cien mil habitantes, siendo conocidas las diferencias de tasas existentes entre los distintos países ${ }^{1}$. Los factores de riesgo se pueden categorizar en dos grupos interdependientes: los factores relacionados con

Recibido: 25/06/2014

Aceptado: 10/09/2014

Este trabajo no cuenta con fuentes de apoyo financiero de ningún tipo.

1 Departamento de Psiquiatría, Escuela de Medicina, Pontificia Universidad Católica de Chile, Santiago, Chile.

2 Alumno de medicina Pontificia Universidad Católica de Chile. 
el individuo y factores relativos a las relaciones sociales y culturales. Entre los factores de riesgo personales clásicamente aceptados se describen: conducta suicida previa, comorbilidad psiquiátrica, abuso de sustancias, sexo masculino, edad avanzada, soltería, entre otros ${ }^{2,3}$. Por otra parte, existirían contextos sociales y culturales que tenderían a correlacionarse en forma directa con factores económicos desfavorables como crisis bursátiles, altas tasas de desempleo ${ }^{4-6}$ y características relativas a la religiosidad. En esta línea, Durkheim ya había propuesto hace más de un siglo que las tasas de suicidio variarían en momentos de inestabilidad económica ${ }^{7}$. Paradojalmente a lo esperado, al considerarlo desde la perspectiva individual, existen algunos estudios que muestran que el desarrollo económico de los países (medido en producto interno bruto per cápita), se acompañaría de un aumento en las tasas de suicidio ${ }^{8}$, e incluso, a nivel local en algunas poblaciones, las tasas de suicidio serían mayores en grupos comparativamente más acomodados que sus pares ${ }^{9,10}$. Estos hallazgos desafían el sentido común y están en aparente contradicción con algunos estudios y reportes, que asocian suicidio con condiciones de vida adversas ${ }^{11}$. Bajo la misma aparente contradicción, el suicidio es difícil de entender desde una perspectiva evolucionaria ya que la conducta suicida atentaría contra el éxito biológico ${ }^{12}$, definido como la capacidad de traspasar los genes a la generación siguiente y que ésta a su vez haga lo mismo. En un intento por explicar esta situación evolucionariamente, se ha asociado la agregación poblacional a una ganancia para la mayoría de los individuos al facilitar el acceso a recursos y la protección, pero al costo de un incremento de la competencia que perjudicará a algunos sujetos más vulnerables ${ }^{13,14}$, e incluso, mediante complejos modelos se ha correlacionado la agregación poblacional con el incremento del riesgo de infecciones proponiéndose el suicidio como una conducta que disminuiría el riesgo de trasmisión de dichas infecciones a la población en desarrollo y que aún no se ha reproducido ${ }^{12}$. Sin embargo, a nuestro entender, la mejoría de un indicador económico, como el producto interno bruto, pudiera asociarse con el suicidio de manera complementaria a otras variables relacionadas al estándar de vida, como lo es el Índice de Desarrollo Humano utilizado por la ONU.

Los propósitos de este trabajo son: verificar el tipo de asociación existente entre las tasas de suicidio y variables asociadas al estándar de vida de los países y proponer una mirada evolucionaria para explicar esta asociación.

\section{Método}

Dada la inmensa cantidad de factores que pueden incidir en el suicidio, decidimos comparar las tasas de suicidio de la mayor cantidad posible de países, con algunos indicadores económicos y de calidad de vida objetivos utilizados por organismos internacionales. Obtuvimos las tasas de suicidio de los diferentes países publicadas en la base de datos de la página web de la Organización Mundial de la Salud (OMS) hasta octubre de $2011^{15}$. Las tasas de suicidio no son publicadas anualmente por todos los países y su año de publicación es variable ${ }^{16}$. Es por esto, que como criterio de inclusión, se requirió tener una tasa de suicidio publicada desde el año 2005 en adelante y que tuvieran publicados indicadores económicos del año 2008. Se eligió el año 2008 para obtener los indicadores económicos, ya que el año promedio de las tasas de suicidio obtenidas se aproximaban a ese año. Fueron excluidos los países que notificaran tasas de suicidio iguales a cero, ya que con alta probabilidad, serían países con registros de salud donde no se notifiquen suicidios como causa de muerte y constituirían un sesgo evitable. En cuanto a los indicadores económicos consideramos la utilización de la paridad de poder de compra (Purchasing Power Parity-PPP) del año $2008^{17}$ ya que es un indicador que permite comparar el producto interno bruto de distintos países ajustando su tipo de cambio según el valor del dólar de ese año. Se utilizó también el índice de desarrollo humano (Human Development Index, HDI) del año $2008^{18}$, desarrollado por las Naciones Unidas, ya que es un índice que relaciona tres dimensiones básicas del desarrollo humano: salud evaluado en esperanza de vida al nacer, educación 
medido como promedio de años de escolaridad junto con años esperados de escolaridad y economía medido como producto interno bruto ajustado por $\mathrm{PPP}^{19}$. La utilización del HDI permite tener una visión del estándar de vida de la población más complejo y profundo de lo que permite la utilización única del PPP. El rango de HDI varía entre 0 y 1 , siendo mayor el desarrollo de las tres dimensiones mientras más se acerque el valor a 1 .

Para el análisis estadístico utilizando el programa SPSS ${ }^{\circledR}$, se realizó la medición de correlaciones no paramétricas de Spearman para comparar tasas de suicidio con PPP y HDI respectivamente. Se consideró como significativo un valor $\mathrm{p}<0,05$.

\section{Resultados}

Un total de 82 países con tasas de suicidio publicadas en la página de estadísticas de la OMS cumplieron los criterios de inclusión. Para la totalidad de esos países obtuvimos el PPP de 2008, en tanto que sólo 78 tenían la información pertinente para el HDI. Con esta información se construyó la Tabla 1.

$\mathrm{Al}$ relacionar las tasas de suicidio globales con los PPP para cada país se obtuvo una correlación positiva (rho $=0,360 ; \mathrm{p}<0,01$ ), lo que se observa en la Figura 1.

Al comparar las tasas de suicidio con HDI también se obtuvo una correlación positiva (rho $=0,366 ; \mathrm{p}<0,01)$, como se muestra en la Figura 2.

Estos datos permiten afirmar que existe una correlación positiva entre tasas de suicidio en los distintos países con PPP y HDI del año 2008.

Tabla 1. Tasas de suicidio de 82 países y relación con paridad del poder de compra (PPP) e Índice de Desarrollo Humano (HDI)

\begin{tabular}{|ccc|}
\hline Indicador & Rho & Valor $\mathrm{p}$ \\
PPP & $+0,360$ & $\mathrm{p}<0,01$ \\
HDI & $+0,366$ & $\mathrm{p}<0,01$ \\
\hline
\end{tabular}

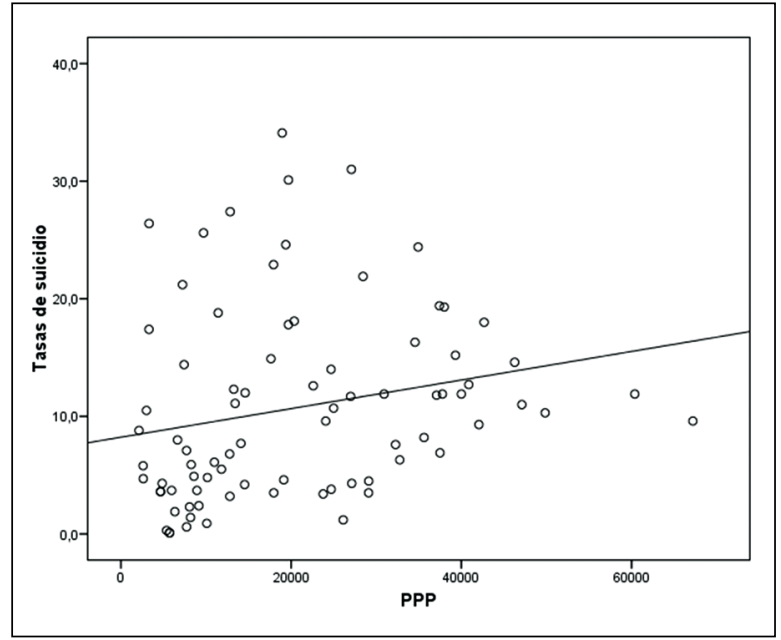

Figura 1. Correlación linear entre tasas de suicidio de 78 países y Paridad del poder de compra (PPP). Rho: 0,360 $(\mathrm{p}<0,01)$.

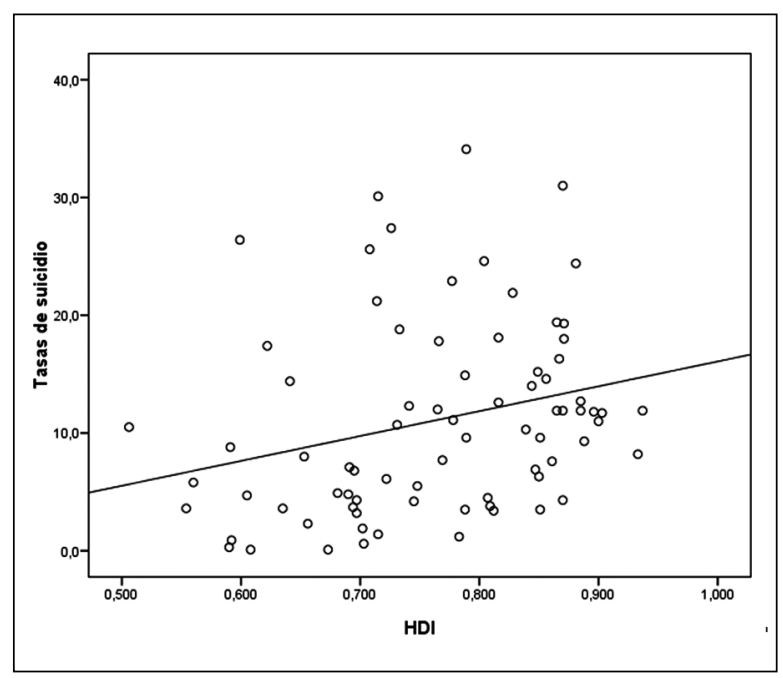

Figura 2. Correlación linear entre tasas de suicidio de 78 países e índice de desarrollo humano (HDI). Rho: 0,366 $(\mathrm{p}<0,01)$.

\section{Discusión}

$\mathrm{Al}$ analizar las tasas de los países pertenecientes a las estadísticas publicados por la OMS, durante la búsqueda impresionó la ausencia de datos sobre este ítem en gran cantidad de países del mundo, siendo los países más desarrollados los con mejo- 
res registros ${ }^{1}$. Sobre este punto cabe hacer mención, que la medición de tasas de suicidio presenta importantes sesgos en relación al registro de datos a nivel mundial. Lo anterior se relaciona con aspectos culturales, religiosos y/o políticos de cada país, donde intervienen a su vez muchas variables difíciles de manejar, como por ejemplo cambios en las condiciones de vida, conflictos internos, etcétera. Estas diferencias varían dependiendo del nivel de desarrollo de los países ${ }^{20}$. Por esa razón, decidimos considerar las tasas de suicidio de la mayor cantidad posible de países, ejercicio que aunque podría tener sesgos de selección, tendería al menos a disminuir parcialmente este sesgo al estudiar una muestra numerosa. Aún así, aunque estamos conscientes de las limitaciones de un trabajo de esta naturaleza, es importante analizar las distintas posibilidades para entender nuestros resultados.

La correlación positiva entre suicidio y algunos indicadores económicos y de calidad de vida observados en este estudio, encuentra antecedentes en contra ${ }^{1,5,21}$ y a favor ${ }^{8,9,16,22-24}$ en publicaciones previas. Pensamos que la mejor manera de abordar este asunto es considerando las siguientes opciones explicativas:

a. Se podría argumentar que los países más ricos tienen una población envejecida en comparación con los países más pobres, en circunstancias que se ha reportado que las tasas de suicidio serían mayores después de los 50 años ${ }^{1,8,25}$. La hipótesis sería que en los países con mayor PPP y HDI la población está más envejecida y que dado que el riesgo de suicidio aumentaría con la edad esa sería la explicación de nuestros resultados. Esto claramente podría ser un factor confundente, sin embargo, hay estudios donde se analizan tasas de suicidios ajustadas por edad y $\mathrm{HDI}^{19}$, donde a pesar del ajuste etario, sigue existiendo una correlación positiva, es decir, en países con mayor ingreso per cápita los mayores de 65 años se suicidan más comparado con los sujetos del mismo grupo etario pero que residen en países con menos recursos. Por otra parte, si se comparan países pertenecientes a la OCDE (Organización para la Cooperación y el Desarrollo Económico), que se podrían considerar relativamente más homogéneos en cuanto a envejecimiento poblacional, además de ser reconocidos por ser los países con mayores ingresos en el mundo y con mejores estadísticas, las muertes por lesiones en cuanto a suicidio y homicidio tienden a aumentar al mejorar los ingresos de estos países a lo largo del tiempo, en comparación con muertes por otro tipo de lesiones como accidentes, caídas, etc., que disminuyen mientras mejores son los ingresos de estos países ${ }^{22}$. Nos parece que estas evidencias nos llevan a abandonar esta hipótesis.

b. Otra expliacación pudiera ser que en los países con mayor PPP, existieran mayores diferencias económicas entre los distintos grupos poblacionales, por lo que al interior de cada país, las tasas de suicidio serían mayores en aquellos con menos recursos y peores condiciones de vida. Esta posibilidad teórica se ve desvirtuada por el hecho de que según los datos del Banco Mundial los índices de desigualdad en general son menores en los países de mayores ingre$\operatorname{sos}^{26}$, sin embargo, se debe considerar como lo han mostrado otros autores, que países con mayores recursos no necesariamente se asocian a una menor mortalidad general si no existe una mejor distribución de los recursos ${ }^{27}$. Young et $\mathrm{al}^{9}$, nos presentan por otra parte, un estudio en que toman todos los suicidios ocurridos la ciudad de Kansas EE.UU., durante un período de cuatro años y los comparan con un grupo control de fallecidos en el mismo condado y los parean por edad, género y raza. Posteriormente revisaron los antecedentes relativos a la situación económica de los individuos y encontraron que en el grupo de suicidas esta era mejor que en aquellos fallecidos por otras causas. Esto es muy relevante ya que estudia el problema en un medio ecológico homogéneo y no sometido a cambios ambientales abruptos, que pudieran ser variables confundentes si estuvieran presentes. En síntesis, existe evidencia de que en determinadas circunstancias la mayor riqueza pudiera tener mayor asociación al suicidio que la pobreza. 
c. Zhang en $1998^{8}$, postuló una teoría del suicidio basado en el incremento poblacional. Sin embargo, en sus datos no se encuentran los países con mayores poblaciones y con mayores tasas de crecimiento demográfico, posiblemente, por la dificultad de obtener las tasas de suicidio en la década en que realizó su estudio. En nuestro estudio observamos que esos países tienen tasas de suicidio en la media o bajo la tasa promedio mundial.

d. Desde la perspectiva evolucionaria, el suicidio se considera una paradoja ya que atentaría contra el éxito reproductivo de la especie, especialmente cuando ocurre antes de que un individuo logre dejar descendencia ${ }^{12}$. De Catanzaro propuso una teoría evolutiva donde el suicidio sería adapatativo si la capacidad de reproducción a futuro de los individuos fuera muy pequeña o si éstos se volvieran una carga para sus descendientes ${ }^{28,29}$. Por otro lado, también se ha propuesto el suicidio como una conducta desadaptativa $^{30}$, lo que está en línea con que el ser humano ha evolucionado para alcanzar la máxima eficiencia en todos los desafíos que implica la supervivencia, la reproducción y la crianza, y todos sus sistemas biológicos, sistémicos y psíquicos están alineados con estas metas. Esto implica entre otras cosas un importante desarrollo de habilidades sociales y sistemas fisiológicos y psíquicos para la obtención y ahorro de recursos de todo tipo. Teniendo esto en cuenta, es posible pensar que desde la perspectiva evolucionaria nuestros hallazgos son compatibles con las siguientes opciones:

1. La debilitación de los vínculos sociales: Nos referimos a la posible disminución de los vínculos sociales de los individuos que pareciera acompañar los procesos de desarrollo económico y social, y que Durkheim atribuyó a los procesos de modernización ${ }^{7}$. Está demostrado que la necesidad de vínculos sociales es el resultado de un largo proceso evolutivo y que la soledad y aislamiento tienden a generar psicopatología $\mathrm{y}$ asociarse a suicidio ${ }^{23,31,32}$.

2. Exceso de abundancia: Ya está dicho que el ser humano ha evolucionado en un contexto de escasez relativa, lo que determina que nuestro funcionamiento biológico está configurado para resolver esa condición ${ }^{33,34}$. Esta afirmación se ve sustentada por la presencia de los circuitos de recompensa y motivación que premian o generan sensación de satisfacción al conseguir logros de importancia biológica: de tipo nutricional, social, reproductivo, etc. ${ }^{35,36}$. Los mismos sistemas, como ocurre con el sabor, tienden a responder con mayor agrado cuando el estímulo sigue un período de privación relativa y lo hace en menor grado cuando la exposición al estímulo es permanente. En esa línea es posible argüir que pudiera ser que para algunas personas, por algunas razones no trasciendan la sensación de satisfacción desde el cumplimiento de necesidades básicas a otras más abstractas pudieran presentar una sensación de pérdida de sentido que sea causa de depresión y suicidio, hipótesis que estaría también en línea con los hallazgos de este estudio. Esta posibilidad es coherente con los estudios que han evidenciado la disminución de la tasa de suicidios en países que viven conflictos bélicos ${ }^{37}$, esto es, cuando la vida está en riesgo el suicidio no es una opción.

En suma, el suicidio es un fenómeno complejo y existen distintas hipótesis que podrían explicar la tendencia a observar un aumento de las tasas de suicidio con el aumento de la calidad de vida. La perspectiva evolucionaria nos ofrece un punto de vista complementario para explicar estos hallazgos que intuitivamente pueden parecer incomprensibles, pero que no lo son al considerarlos de esta manera. Sin embargo, son necesarios otros estudios para poder apoyar esta hipótesis, ya que los datos en suicidio tienen limitaciones estadísticas conocidas a lo que se suman las limitaciones de los estudios ecológicos en que al no ser posible individualizar los datos caso a caso se puede caer en una falacia ecológica. Es por esto, que es fundamental mejorar la calidad de los registros a nivel mundial, así como también, la investigación en el tema para poder entender de mejor manera el fenómeno del suicidio y así lograr mejores formas de prevención y manejo. 


\begin{abstract}
Resumen
Introducción: El suicidio es un fenómeno complejo y evolucionariamente en apariencia paradojal, siendo conocidos los múltiples factores de riesgo asociados y las diferencias de tasas existentes entre los países. Comúnmente se atribuye mayor riesgo a dificultades económicas y peor calidad de vida, sin embargo, algunos estudios mostrarían una tendencia inversa. Objetivo: Comparar las tasas de suicidio de distintos países publicadas hasta la fecha, con algunos indicadores económicos y de calidad de vida objetivos. Métodos: Se correlacionaron las tasas de suicidio de 82 países publicados por la Organización Mundial de la Salud, con la paridad de poder de compra $(P P P)$ e indice de desarrollo humano (HDI) del año 2008 publicados por el Banco Mundial y las Naciones Unidas respectivamente. Resultados: Se obtuvo una correlación positiva entre tasas de suicidio con PPP e HDI ( $r=0,3601$ y $r=0,366$ respectivamente, $p<0,01)$. Conclusión: La correlación positiva observada, es decir, mientras mejores indicadores económicos y de calidad de vida, habría mayores tasas de suicidio, son en apariencia contradictorios. Proponemos que la comprensión de estos datos desde una perspectiva evolucionaria podría contribuir a un mejor entendimiento de éstos. Sin embargo, son múltiples los factores que podrían estar involucrados en este resultado, por lo que es necesaria mayor investigación para poder entender de mejor manera el fenómeno del suicidio a nivel mundial.
\end{abstract}

Palabras clave: Suicidio, Desarrollo Económico, Evolución Biológica, Calidad de vida.

\section{Referencias bibliográficas}

1. Hawton K, Van Heeringen K. Suicide. Lancet 2009; 373: 1372-81.

2. Sudak H. Suicide. In B.J. Sadock, V. Sadock \& P. Ruiz (Eds) Kaplan \& Sadock's Comprehensive Textbook of Psychiatry, 9th edition, Lippincott Williams \& Wilkins 2009; pp. 2717-31.

3. Zhuoyang L, Page A, Martin G, Taylor R. Attributable risk of psychiatric and socio-economic factors for suicide from individual-level, populationbased studies: A systematic review. Soc Sci Med 2011; 72: 608-16.

4. Lamar J. Suicides in Japan reach a record High. BMJ 2000; 321: 528.

5. Ceccherini-Nelli A, Priebe S. Economic factors and suicide rates: associations over time in four countries. Soc Psychiat Epidemiol 2010; 46: 975-82.

6. Kim H, Song YJ, Yi JJ, Chung WJ, Nam CM. Changes in mortality after the recent economic crisis in South Korea. Ann Epidemiol 2004; 14: 442-6.

7. Durkheim E. Le Suicide: Étude de sociologie. Paris: Alcan. 1897.

8. Zhang J. Suicide in the world: toward a population increase theory of suicide. Death Studies 1998; 22: 525-39.

9. Young T, Wooden S, Dew P, Hoff G, Cai J. The
Richard Cory phenomenon: suicide and wealth in Kansas City, Missouri. J Forensic Sci 2005; 50: 2.

10. Panczak R, Galobardes B, Voorpostel M, Spoerri A, Zwahlem M, Egger M; Swiss National Cohort and Swiss Household Panel. A Swiss neighbourhood index of socioeconomic position: development and association with mortality. J Epidemiol Community Health 2012; 66: 1129-36.

11. Stack S. Suicide: A 15 year review of the sociological literature. Part 1: cultural and economic factors. Suicide Life Threat Behav 2000; 30: 145-62.

12. Tanaka M, Kinney DK. An evolutionary hypothesis of suicide: why it could be biologically adaptive and is so prevalent in certain occupations. Psychol Rep 2011; 108: 977-92.

13. De Catanzaro D. Suicidal Ideation and the Residual Capacity to Promote Inclusive Fitness: A Survey. Suicide and Life-Threat Behavi 1984; 14: 75-87. doi: 10.1111/j.1943-278X.1984.tb00339.x

14. Nonaka E, Parvinen K, Brännström A. Evolutionary suicide as a consequence of runnaway selection for grater aggregation tendency. J Theor Biol 2013; 317: 96-104.

15. Programa de salud mental OMS, disponible en URL: http://www.who.int/mental_health/prevention/suicide/country_reports/en/index.html

16. Blasco-Fontecilla H, Pérez-Rodríguez M, García- 
Nieto R, Fernández-Navarro P, Galfavy H, de León J, et al. Worlwide impact of economic cycles on suicide trends over 3 decades: differences according to level of development. A mixed effect model study. BMJ Open 2012; 2: e000785 doi: 10.1136/bmjopen-2011-000785.

17. Estadísticas del Banco Mundial, disponibles en URL: http://data.worldbank.org/indicator/NY. GNP.PCAP.PP.CD

18. International human develpment indicators $\mathrm{UN}$, disponible en URL: http://hdr.undp.org/en/data/ profiles/

19. Shah A. The relationship between elderly suicide rates an the human development index: a crossnational study of secondary data from the World Heatlh Organization and the United Nations. International Psychogeriatrics 2009; 21: 69-77.

20. Vijayakumar L, Nagaraj K, Pirkis J, Whiteford H. Suicide in developing countries: frequency, distribution and association with socioeconomic indicators. Crisis 2005; 26: 104-11.

21. Kalist DE, Molinari NA, Siahaan F. Income, employment and suicidal behavior. J Ment Health Policy Econ 2007; 10: 177-87.

22. Moniruzzaman S, Andersson R. Economic development as a determinant of injury mortality-A longitudinal approach. Soc Sci Med 2008; 66: 1699-708.

23. Moyano E, Barría R. Suicidio y producto interno bruto (PIB) en Chile: hacia un modelo predictivo. Rev Latin Psicol 2006; 38: 343-59.

24. Bando DH, Moreira RS, Pereira JC, Barrozo LV. Spatial clusters of suicide in the municipality of Sao Paulo 1996-2005: an ecological study. BMC Psychiatry 2012; 12: 124.

25. Shah A. A replication of the relationship between elderly suicide rates and human development indez in a cross-national study. International Psychogeriatrics 2010; 22: 727-32.

26. Dyer O. Inequality is barrier to global development, says World Bank. BMJ 2005; 24: 331.

27. Biggs B, King L, Basu S, Stuckler D. Is wealthier always healthier? The impact of national income level, inequality, and poverty on public health in Latin American. Soc Sci Med 2010; 71: 266-73.

28. De Catanzaro D. Human suicide: a biological perspective. Behavioral and Brain Science 1980; 3: 265-90.

29. De Catanzaro D. Suicide and self-damaging be- havior: a sociobiological perspective. New York: Academic Press. 1981.

30. Saad G. Suicide triggers as sex-specific threats in domains of evolutionary import: negative correlation between global male-to-female suicide ratios and average per capita gross national income. Med Hypotheses 2007; 68: 692-6.

31. Kelly BD, Davoren M, Mhaoláin AN, Breen EG, Casey P. Social capital and suicide in 11 European countries: an ecological analysis. Soc Psychiatry Psychiatr Epidemiol 2009; 44: 971-7.

32. Compton MT, Thompson NJ, Kaslow NJ. Social environment factors associated with suicide attempt among low-income African Americans: the protective role of family relationships and social support. Soc Psychiatry Psychiatr Epidemiol 2005; 40: $175-85$.

33. Eaton SB, Konner M, Shostak M. Stone agers in the fast lane; chronic degenerative diseases in evolutionary perspective. Am J Med 1988; 84: 739-49.

34. Eaton SB, Strassman BI, Nesse RM, Neel JV, Ewald PW, Williams GC, et al. Prev Med 2002; 34: 10918.

35. Yamamoto T. Central mechanisms of roles of taste in reward and eating. Acta Physiol Hung 2008; 95 (2): 165-86.

36. Carlezon WA Jr, Thomas MJ. Biological substrates of reward and aversion: a nucleus accumbens activity hypothesis. Neuropharmacology 2009; 56 Suppl 1: 122-32.

37. Selakovic-Bursic S, Haramic E, Leenaars AA. The Balkan Piedmont: male suicide rates pre-war, wartime, and post-war in Serbia and Montenegro. Arch Suicide Res 2006; 10: 225-38.

Correspondencia:

Dr. Jaime Santander Toro

27548873

Departamento de Psiquiatría

Clínica UC San Carlos de Apoquindo

Av Camino El Alba 12.351

Las Condes

Santiago, Chile.

Zip Code: 7620002

E-mail: jsantan@med.puc.cl 